\title{
Heat shock protein 27, a novel regulator of 5 -fluorouracil resistance in colon cancer
}

\author{
MASASHI TSURUTA ${ }^{1}$, HIDEKI NISHIBORI ${ }^{1,3}$, HIROTOSHI HASEGAWA ${ }^{1}$, YOSHIYUKI ISHII ${ }^{1}$, \\ TAKASHI ENDO ${ }^{2}$, TETSURO KUBOTA ${ }^{2}$, MASAKI KITAJIMA $^{4}$ and YUKO KITAGAWA ${ }^{1}$ \\ ${ }^{1}$ Department of Surgery and ${ }^{2}$ Center for Comprehensive and Advanced Medicine, Keio University, 35 Shinanomachi, \\ Shinjuku-ku, Tokyo 160-8582; ${ }^{3}$ Kitami Gastrointestinal Clinic, 8-10-8 Kitami, Setagaya-ku, Tokyo 157-0067; \\ ${ }_{4}^{4}$ International University of Health and Welfare, Mita Hospital, 1-4-3 Mita, Minato-ku, Tokyo 108-8309, Japan
}

Received May 5, 2008; Accepted July 7, 2008

DOI: 10.3892/or_00000125

\begin{abstract}
The resistance of colon cancer to 5-fluorouracil (5-FU) is a critical issue, and the cause of this resistance cannot always be explained based on the known molecules. Heat shock protein 27 (HSP27) mRNA expression has recently been shown to be correlated with 5-FU resistance in 5-FU-resistant cell lines. In this study, we attempted to elucidate the functional mechanism of HSP27 in 5-FU resistance in colon cancer. HSP27 protein levels in several human colon cancer cell lines (LoVo, HCT15, WiDr, HCT116, HT-29 and SW480) were determined by immunoblot and densitometry analysis. The in vitro growth inhibition rates (IR) of the cell lines at various concentrations of 5-FU were assessed by MTT assay. The degree of 5-FU resistance was estimated as the drug concentration inducing 50\% IR $\left(\mathrm{IC}_{50}\right)$. The HSP27 protein level and $\mathrm{IC}_{50}$ were significantly correlated in these cell lines $(\mathrm{p}=0.010)$. The effect of HSP27 overexpression on $\mathrm{IC}_{50}$ was evaluated in LoVo cells. HSP27 transfectants significantly increased $\mathrm{IC}_{50}$ and reduced HSP27 resistance. The effect of HSP27 down-regulation by HSP27 siRNA on $\mathrm{IC}_{50}$ was confirmed in HCT15 cells. HSP27 siRNA suppressed HSP27 protein levels and reduced $\mathrm{IC}_{50}$ in a dose-dependent manner. These data indicated that HSP27 is closely connected with 5-FU resistance in colon cancer and suggested that HSP27
\end{abstract}

Correspondence to: Dr Masashi Tsuruta, Department of Surgery, Keio University, 35 Shinanomachi, Shinjuku-ku, Tokyo 160-8582, Japan

E-mail: championtsuru@yahoo.co.jp

Abbreviations: 5-FU, 5-fluorouracil; HSP27, heat shock protein 27; IR, inhibition rates; HSP, heat shock protein; sHSPs, the small heat shock proteins; MTT, 2,3-bis (2-methoxy-4-nitro-5-sulfophenyl)2H-tetrazolium-5-carboxanilide inner salt; hRFI, the human ring finger homologous to inhibitor of apoptosis protein type

Key words: heat shock protein 27, 5-fluorouracil, colon cancer, RNA interference levels predicted 5-FU resistance. HSP27 down-regulation overcame 5-FU resistance and HSP27 may be a clinical target in patients with 5-FU-resistant colon cancer.

\section{Introduction}

5-Fluorouracil (5-FU), first synthesized by Heidelberger et al, in 1957, has been employed for the treatment of various solid cancers for $>40$ years (1). Currently, combinations of 5-FU with other chemotherapeutic agents such as irinotecan and oxaliplatin have improved the response rate and survival for advanced colorectal cancer (2). However, inherent or acquired resistance to 5-FU therapy is still a critical problem. Various studies have reported some determinants of resistance to 5-FU, and various mechanisms have been proposed, including overexpression of the target enzyme, thymidylate synthase, depletion of folate cofactors, an increase in the level of the competing substrate deoxyuridine monophosphate, altered drug transport/metabolism, and alterations in DNA repair/cellcycle control pathways (3-7). Aschele et al reported that the immunohistochemical quantitation of thymidylate synthase expression in colorectal cancer metastases was a predictor for clinical response to 5-FU-based chemotherapy (5). Zhou et al demonstrated that dihydropyrimidine dehydrogenase activity in the blood can be used to predict the toxicity and 5-FU plasma concentration in colorectal cancer patients undergoing 5-FU-based chemotherapy (8). However, there are no reliable biomarkers for clinical use in 5-FU-based chemotherapy. To screen the candidate genes expressed in association with 5-FU resistance, Takechi et al performed an mRNA differential display and Northern blot analysis with three pairs of parent/5-FU-resistant human gastrointestinal tumor cell lines (9). The analysis revealed 20 overexpressed and 10 underexpressed genes in at least one of three 5-FUresistant cells when compared with their parent cells. Heat shock protein 27 (HSP27) was found to be overexpressed in HT-29/5-FU resistant cells by Northern hybridization.

Under physiological conditions, cytoplasmic heat shock proteins (HSPs) play an important role as molecular chaperones in protein-protein interactions, including translocation, folding and the prevention of inappropriate protein aggregation. Five major families of HSPs have been identified that differ in size, 
structure and function. Specifically, these families of HSPs are comprised of HSP110, HSP90, HSP70, HSP60 and the small heat shock proteins (sHSPs). HSPs are constitutively expressed at low levels in the cytoplasm of many human cells, and their expression levels increase following exposure to a wide range of stimuli, such as elevated temperature, toxins, infection, inflammation or ischemia (10-12). Furthermore, correlation of HSP expression with a clinical response such as a drug or radiation resistance can be expected (13). There is considerable in vitro evidence showing that overexpression of HSP27 in tumor cells enhances their tumorigenicity and protects against cell death triggered by a number of stimuli. It is unclear how the HSPs become overexpressed in cancer. Ciocca et al supposed that the physiopathological features of the tumor microenvironment (low glucose, $\mathrm{pH}$ and oxygen) tended toward HSP induction (14). HSP27 and HSP70 may also be involved in anti-cancer drug resistance (15-17). HSP27 was found to participate in the resistance of colorectal cancer cells to doxorubicin (18). Expression of HSP27 and HSP70 has been associated with drug resistance in breast cancer patients treated with relatively high doses of anthracyclines (19). However, there have been no reports on the relationship between HSP27 and 5-FU resistance in colorectal cancer.

Takechi et al (9) supported the possible relationship of HSP27 to 5-FU resistance in colon cancer. If a correlation between HSP27 and 5-FU resistance in colon cancer can be elucidated, HSP27 may be a novel clinical biomarker and a good target of gene therapy for 5-FU resistance. With this background in mind, our aim was to determine the in vitro functional correlation between 5-FU resistance and HSP27 protein levels in colon cancer.

\section{Materials and methods}

Drug. 5-FU was purchased from Kyowa Hakko Kogyo Co. Ltd., Tokyo.

Cell lines. The human colon cancer cell lines SW480, WiDr, HT-29, HCT15, HCT116 and LoVo, were obtained from the American Type Culture Collection (Rockville, MD). SW480 and WiDr were grown in Dulbecco's modified Eagle's medium, and the others in Roswell Park Memorial Institute1640 medium (Gibco, MA). Each was supplemented with $10 \%$ fetal bovine serum (CSL Ltd., Australia) and 1\% penicillin/ streptomycin. The cells were cultured at $37^{\circ} \mathrm{C}$ with $5 \% \mathrm{CO}_{2}$.

Cell proliferation assay. The cell proliferation assay was carried out using the 2,3-bis (2-methoxy-4-nitro-5-sulfophenyl)-2H-tetrazolium-5-carboxanilide inner salt (MTT) assay as described previously (20). Briefly, 5,000 cells per well in 96-well microtiter plates (Sumilon, Sumitomo Bakelite Co., Tokyo, Japan) were incubated for $24 \mathrm{~h}$, exposed to various concentrations of 5-FU for $48 \mathrm{~h}$ continuously and thereafter to a drug-free medium for another $24 \mathrm{~h}$. The absorbance in the wells was measured with an NJ-2300 microplate spectrophotometer at 540 and $630 \mathrm{~nm}$ (Immuno Reader, Nalgen Nunc International, Rochester, NY). The inhibition rate (IR) was calculated using the equation: [1-(mean absorbance of drug well/mean absorbance of control wells)]x $\sim 100(\%)$. Absorbance of each experimental well was adjusted by the mean absorbance of blank wells. 5-FU resistance was evaluated by $\mathrm{IC}_{50}$, which was determined as the concentration of the drug required to inhibit cell growth by $50 \%$ relative to the untreated cells.

Western blot analysis for HSP27. Total cell lysates were extracted with lysis buffer as reported previously (21). The quantity of cell lysates was determined using a Bio-Rad DC protein assay kit (Bio-Rad Laboratories, Hercules, CA) and $20 \mu \mathrm{g}$ of lysates in total were resolved in Readygela $\mathrm{J}$ (BioRad Laboratories) and transferred to an Immuno-Blot ${ }^{\text {TM }}$ polyvinylidene fluoride membrane (Bio-Rad Laboratories). The membrane was blocked in phosphate-buffered saline containing $5 \%$ non-fat milk powder for $2 \mathrm{~h}$ at room temperature, and then incubated at $4^{\circ} \mathrm{C}$ overnight with 1:2000diluted anti-human HSP27 mouse monoclonal antibody (G3.1, Lab Vision Corporation, CA), 1:1000-diluted anti-human HSP27 rabbit polyclonal antibody (Stressgen, BC, Canada) or 1:5000-diluted anti-human, ß-actin mouse monoclonal antibody (AC74, Sigma, MO). Membranes were incubated for $30 \mathrm{~min}$ with a 1:5000-diluted horseradish peroxidaseconjugated anti-mouse IgG (Promega Corporation, WI) or anti-rabbit IgG (Stressgen). Bound complexes were detected using the ECL-Plus reagent (Amersham Biosciences Corporation, Cardiff, UK) according to the manufacturer's instructions. Density of the band was measured using NIH image (Scion) software, and normalized by B-actin. Each experiment was performed in triplicate.

Transfection with HSP27 sense expression vector. We obtained the HSP27 sense expression vector, pTracer-SV40HSP27 from K. Yamamoto, Asahi Breweries, Ltd. (22). The empty vector pTracer-SV40 was purchased from InvitrogenLife Technologies, Inc., CA. Transfection was performed according to the method described previously (22).

Briefly, LoVo cells were plated at $5.0 \times 10^{4}$ cells in $6 \mathrm{~cm}$ plates. After $24 \mathrm{~h}$ incubation, the cells were rinsed with OptiMEM (Invitrogen-Life Technologies, Inc.) and $1 \mathrm{ml}$ of OptiMEM containing $15 \mu \mathrm{l}$ of lipofectamine 2000, a cationic lipid (Invitrogen-Life Technologies, Inc.), and $5 \mu \mathrm{g}$ of expression vector were added. After incubation for $5 \mathrm{~h}$ at $37^{\circ} \mathrm{C}$, the medium was replaced with the regular medium described above. Transfectants were further incubated for $24 \mathrm{~h}$ and evaluated for HSP27 protein level and 5-FU resistance. We used recombinant HSP27 protein, which was purchased from Stressgen, to confirm HSP27 expression.

HSP27 siRNA. The sequence of HSP27 siRNA corresponded to the human HSP27 site (5'-UAGCCAUGCUCGUCCUGC CUU-3'). The sequence of the scrambled siRNA duplex was (5'-ACGUAAGGCGCGUAACGGGTT-3'). RNA interference was performed using lipofectamine 2000 according to the manufacturer's instructions (Invitrogen-Life Technologies, Inc.). Twenty-four hours before treatment with siRNA, HCT15 cells were plated at $2.0 \times 10^{4}$ on $6 \mathrm{~cm}$ plates

Cells were treated with various concentrations of siRNA after preincubation for $20 \mathrm{~min}$ with $20 \mu 1$ of lipofectamine 2000 in serum-free OptiMEM (Invitrogen-Life Technologies, Inc.). Four hours after the start of incubation, the medium was replaced with a regular culture medium as described 
$\mathbf{A}$

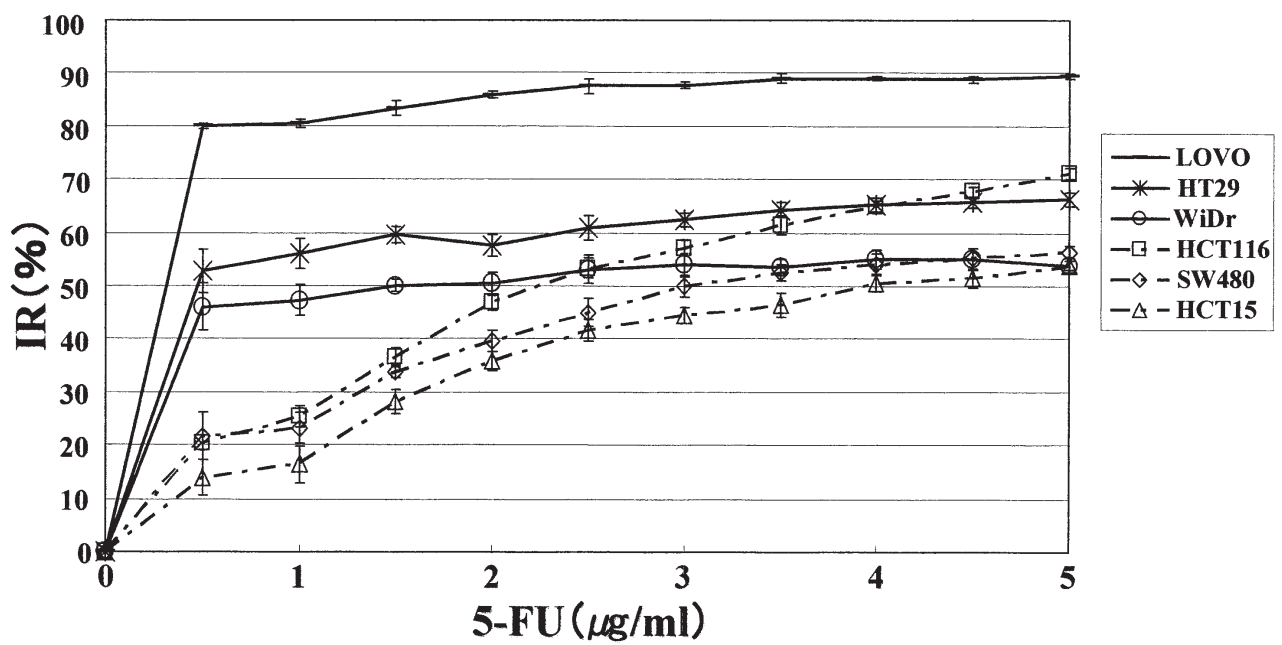

B

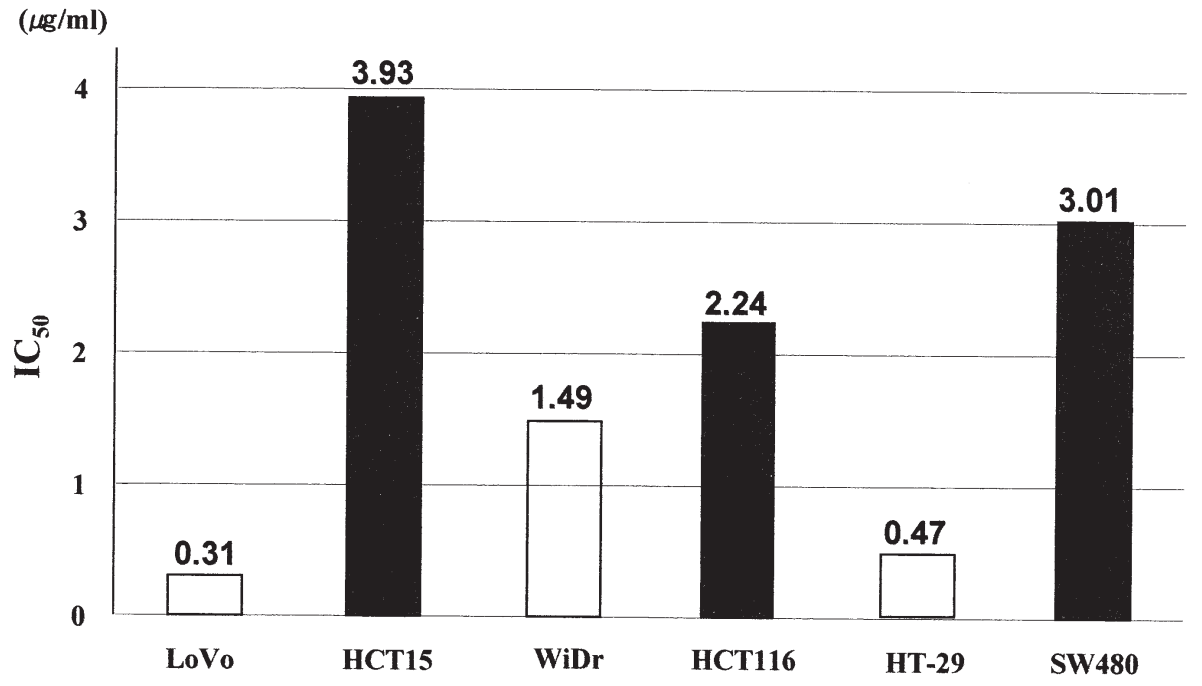

Figure 1. 5-FU resistance of human colon cancer cell lines. (A) Cells were exposed to various concentrations of 5-FU for 2 days, and the growth inhibition rate (IR) was assessed by MTT assay. Points indicate the analysis in triplicate and bars, SD. (B) The degree of resistance was estimated by the drug concentrations that caused $50 \%$ growth inhibition as compared with the control $\left(\mathrm{IC}_{50}\right)$.

above. Transfectants were incubated for $24 \mathrm{~h}$ and evaluated for 5-FU resistance and HSP27 protein levels.

Statistical analysis. Each value was expressed as mean \pm standard deviation. Statistical analysis was performed by the Mann-Whitney U test or regression analysis using the software, Statistical Package for the Social Sciences 13.0J for Windows (SPSS, Chicago, IL). $\mathrm{P}<0.05$ was considered as significant.

\section{Results}

5-FU resistance of human colon cancer cell lines. We first determined 5-FU resistance of 6 human colon cancer cell lines (LoVo, HCT15, WiDr, HCT116, HT-29 and SW480). The cell growth inhibition by 5-FU was analyzed using the MTT assay, and 5-FU resistance was determined by $\mathrm{IC}_{50}$ (Fig. $1 \mathrm{~A}$ and B). Based on these data, three lines (HCT15, SW480 and HCT116) showed high $\mathrm{IC}_{50}$ values $(3.93,3.01$ and $2.24 \mu \mathrm{g} / \mathrm{ml}$, respectively), while the others (LoVo, WiDr and HT-29) showed low values $(0.31,1.49$ and 0.47 , respectively). The former cell lines can be categorized as resistant to 5-FU and the latter as sensitive to 5-FU.

Correlation between HSP27 protein level and 5-FU resistance. Western blot analysis was carried out to determine HSP27 protein levels (Fig. 2A). 5-FU-resistant cell lines, HCT15, SW480 and HCT116 produced high HSP27 protein levels $(2.78,2.52$ and 2.43 , respectively), while in the 5-FU sensitive cell lines, LoVo, WiDr and HT-29, the HSP27 protein levels were low $(0.05,0.28$ and 0.15 , respectively). There was a significant correlation between the HSP27 protein levels and $\mathrm{IC}_{50}$ in these cell lines ( $\mathrm{p}=0.010$, Fig. 2B).

HSP 27 transfectants acquired 5-FU resistance. To determine whether HSP27 overexpression induced resistance to 5-FU, we transiently transfected the pTracer-SV40-HSP27 (HSP27 transfectants) or the empty vector, pTracer-SV40 (mock transfectants) into the 5-FU sensitive colon cancer cell line, LoVo, and evaluated 5-FU resistance. As shown in Fig. 3A, HSP27 transfectants were confirmed to produce HSP27 
A

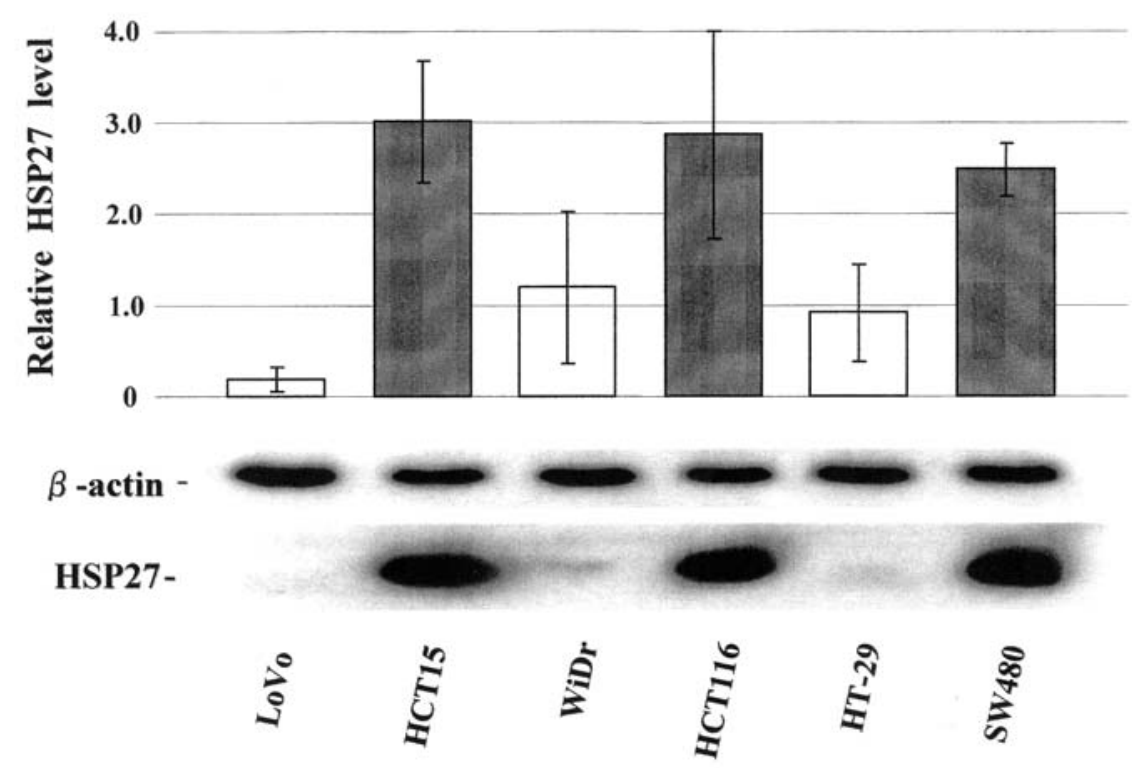

B

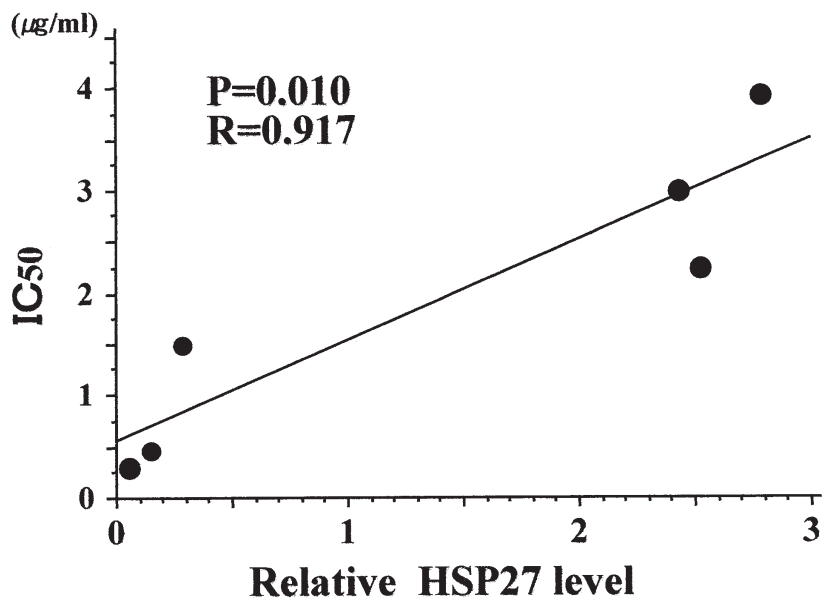

Figure 2. Correlation between HSP27 protein level and $\mathrm{IC}_{50}$ of colon cancer cell lines. (A) HSP27 protein levels of the cell lines were determined by densitometry of Western blot analysis and standardized by $ß$-actin levels. (B) Correlation between the HSP27 protein levels and $\mathrm{IC}_{50}$ of colon cancer cell lines was evaluated by regression analysis.

protein by Western blot analysis. The $\mathrm{IC}_{50}$ in HSP27 transfectants was significantly higher than that in mock transfectants $(\mathrm{p}<0.01$, Fig. $3 \mathrm{~B}$ and C).

HSP27 siRNA. We tested whether the down-regulation of HSP27 affected 5-FU resistance. The 5-FU-resistant cell line HCT15 was transfected with several concentrations (1, 10 and $100 \mathrm{nM}$ ) of HSP27 siRNA or $100 \mathrm{nM}$ scrambled control, and analyzed for HSP27 protein levels and 5-FU resistance. As shown in Fig. 4A, treatment of HCT15 with siRNA reduced HSP27 protein levels in a dose-dependent manner. To determine whether the down-regulation of HSP27 in HCT15 cells overcame 5-FU resistance, the $\mathrm{IC}_{50}$ of each transfectant was evaluated by the MTT assay. Fig. 4B and C showed that
HSP27 siRNA suppressed 5-FU resistance of HCT15 in a dose-dependent manner. There was a significant correlation between HSP27 protein levels and $\mathrm{IC}_{50}$ (p=0.039, Fig. 4D).

\section{Discussion}

5-FU-based chemotherapy has remarkably improved the prognosis of colorectal cancer since new drugs such as oxaliplatin and irinotecan were developed (2). However, inherent or acquired resistance to 5-FU is still a critical issue. A new determinant is required to elucidate the mechanisms of 5-FU resistance, which may lead to overcoming 5-FU resistance and improving survival. Takechi et al proposed HSP27 as a potential determinant of 5-FU resistance in their comprehensive study (9). No previous reports have indicated that 5-FU resistance was influenced by HSP27 in colon cancer. We attempted to elucidate the functional correlation between HSP27 and 5-FU resistance in this study.

We found a significant correlation between HSP27 protein levels and 5-FU resistance in the colon cancer cell lines of LoVo, HCT15, WiDr, HCT116, HT-29 and SW480 (Fig. 2B). It appeared that HSP27 was involved in inherent 5-FU resistance in colon cancer. This was a novel finding for HSP27 although it had been reported to cause resistance to other drugs in several types of cancer $(15,19,22)$. However, the functional correlation between HSP27 and 5-FU resistance in colon cancer was unclear.

To determine whether a change of HSP27 protein levels actually affects 5-FU resistance in colon cancer cell lines, we transiently transfected the HSP27 expression vector into the LoVo cell line, and analyzed changes in 5-FU resistance. 5-FU resistance was significantly enhanced in the HSP27 transfectant (Fig. 3C), which strongly suggested that HSP27 was a key functional molecule in 5-FU resistance.

We also transfected HSP27 siRNA into HCT15 and evaluated the effects of HSP27 down-regulation on 5-FU resistance. HSP27 down-regulation reduced 5-FU resistance in a dose-dependent manner as expected (Fig. 4C and D). 
A

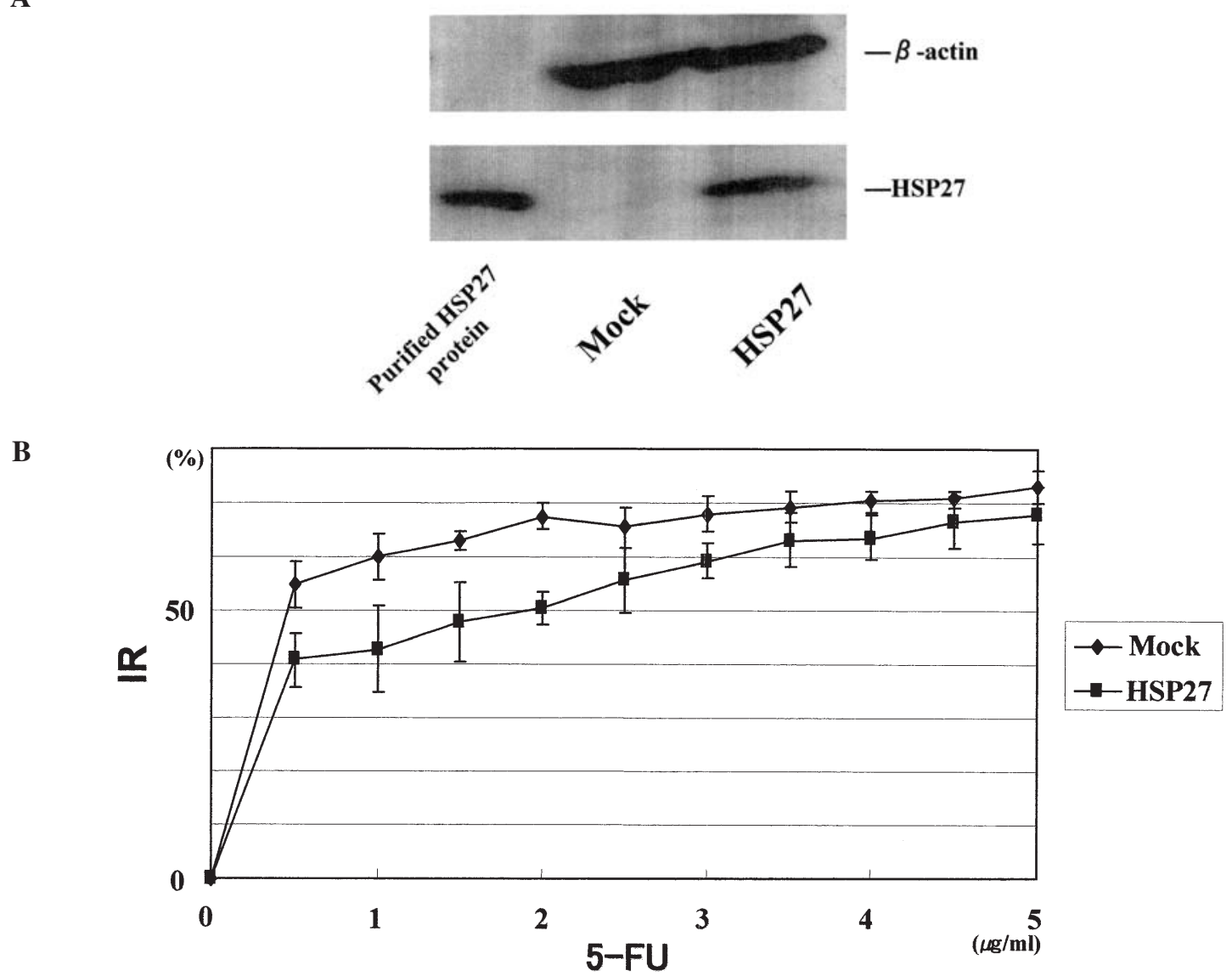

C

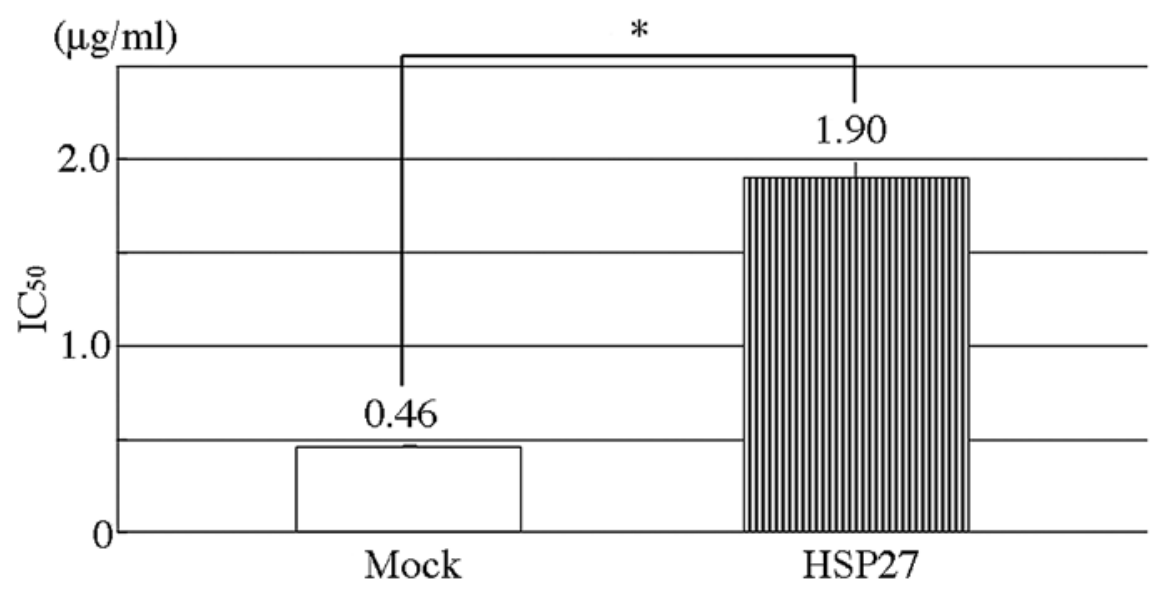

Figure 3. Overexpression of HSP27 reduced 5-FU resistance in the colon cancer cell line LoVo, which was transiently transfected with the pTracer-SV40 (mock) and pTracer-HSP27. (A) One day after transfection, proteins were exacted from transfected cells, and HSP27 and B-actin protein levels were analyzed by Western blot analysis. (B) Transfectants were exposed to various concentrations of 5-FU for 2 days and cell viability was evaluated by MTT assay. (C) 5-FU resistance was evaluated by $\mathrm{IC}_{50}$, as described above. ${ }^{*} \mathrm{P}<0.01$ by Mann-Whitney $\mathrm{U}$ test.

Collectively these findings suggested that HSP27 protein levels were closely related to 5-FU resistance in colon cancer and that HSP27 is one of the major determinants of 5-FU resistance. Although these are in vitro data, this may be a first step toward applying HSP27 clinically in conjunction with 5-FU-based chemotherapy for colon cancer. 5-FU resistance may be predicted by HSP27 levels in colon cancer tissue using immunohistochemistry or real-time polymerase chain reaction. The predicted resistance may lead to a safe and efficiently personalized treatment. Furthermore, HSP27 down-regulation may provide a novel insight into 5-FU-based chemotherapy. We used RNA interference for HSP27 downregulation, and succeeded in overcoming 5-FU resistance. RNA interference has recently drawn considerable attention, and HSP27 siRNA may be applied in gene therapy. Our findings revealed not only a correlation between HSP27 and 5-FU resistance but also the possibility of HSP27-targeted gene therapy. 
$\mathbf{A}$

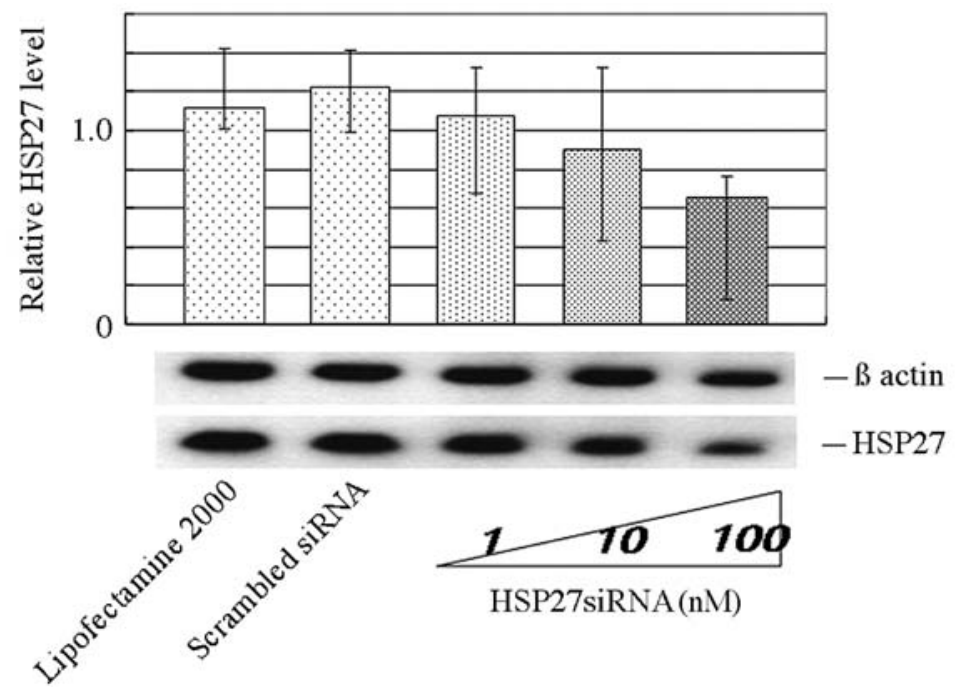

B

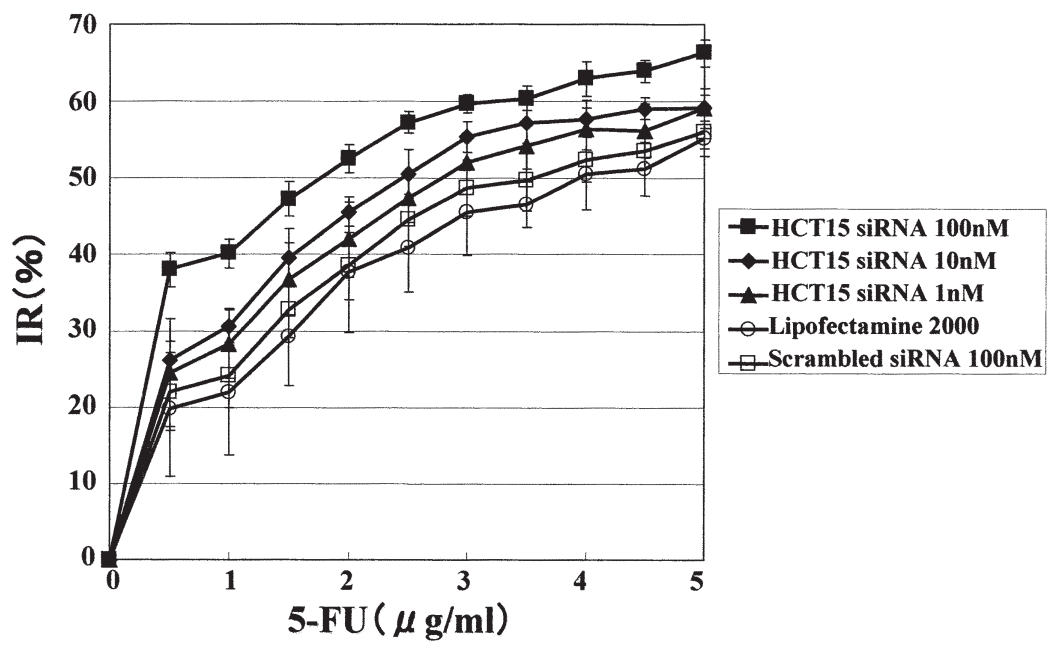

C

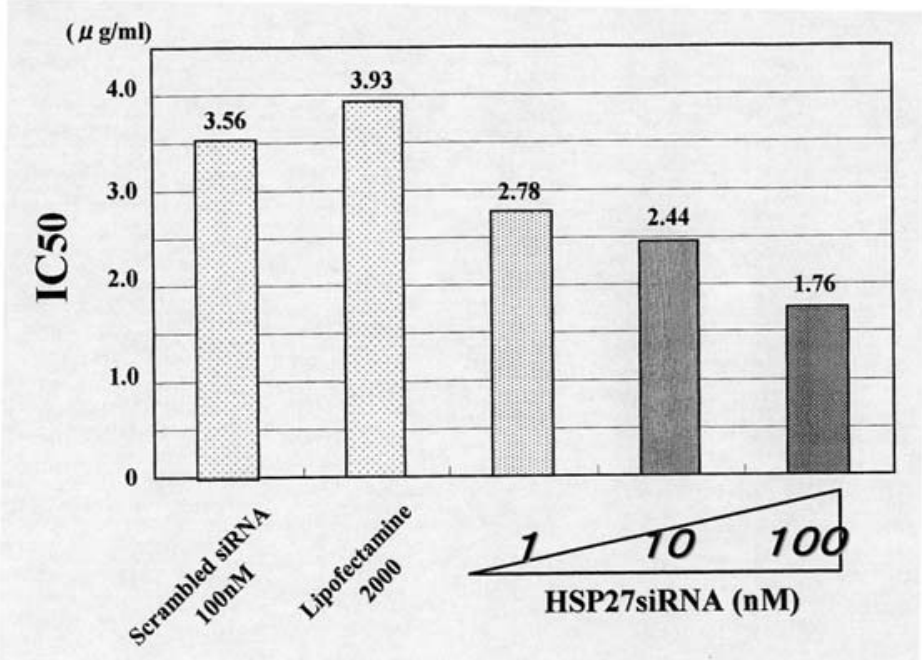

D

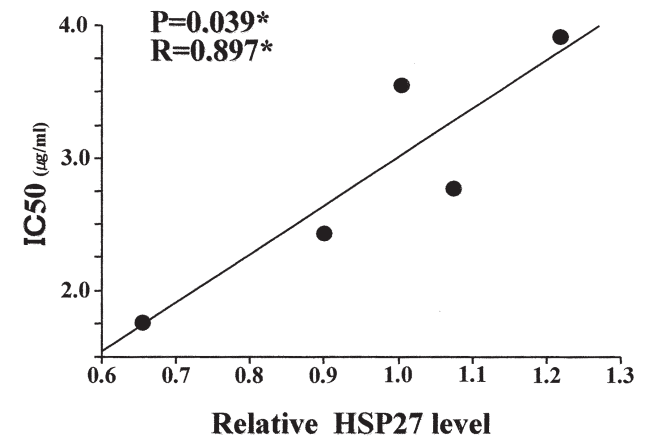

Figure 4. Dose-dependent inhibition of HSP27 protein levels by HSP27 siRNA in HCT15. The colon cancer cell line HCT15 was treated with various concentrations of HSP27 siRNA, its scrambled control, or lipofectamine 2000 for only one day. (A) One day after treatment, proteins were exacted from culture cells, and HSP27 and B-actin protein levels were analyzed by Western blot analysis. (B) Transfectants were exposed to various concentrations of 5-FU for 2 days and cell viability was evaluated by MTT assay. (C) 5 -FU resistance was evaluated by $\mathrm{IC}_{50}$. (D) Correlation between HSP27 protein levels and $\mathrm{IC}_{50}$ in $\mathrm{HCT} 15$ treated with siRNA was evaluated by regression analysis. 
Inducing apoptosis is one of the main mechanisms by which 5-FU eliminates cancer cells (23-25). Resistance to the induction of apoptosis is one of the most important determinants of chemoresistance to 5-FU (26-28). Therefore, elucidation of the mechanism of cellular resistance to 5-FUinduced apoptosis is a critical issue requiring resolution to improve the survival of patients with advanced colorectal cancer. 5-FU induces apoptosis in colorectal cancer cells predominantly through the mitochondrial apoptotic pathway, which involves the release of cytochrome $\mathrm{c}$, and subsequent activation of the upstream initiator caspase- 9 and the downstream effector caspase-3 (29-33). Konishi et al recently revealed that overexpression of the human ring finger homologous to inhibitor of apoptosis protein type (hRFI) in colon cancer cell lines resulted in resistance to $5-\mathrm{FU}$ with the activation of NF- $\mathrm{KB}$ and up-regulation of Bcl-2 and Bcl-XL (34). They suggested that hRFI may be a novel therapeutic target for gene therapy in colorectal cancer. Similarly, HSP27 functions as a negative regulator of cytochrome c-dependent activation of procaspase-3. Concannon et al reported that HSP27 inhibits cytochrome c-mediated caspase activation by sequestering procaspase- 3 and cytochorome c (35). Therefore, HSP27 is a potential determinant of 5-FU resistance in colorectal cancer through its effect on the regulation of apoptosis. The relationship between HSP27 and hRF1 is still unknown and its elucidation is needed.

We confirmed that HSP27 is a key molecule of 5-FU resistance in colon cancer, but the correlation between HSP27 and the other known molecules such as thymidylate synthase or dihydropyrimidine dehydrogenase remain unclear. The difference in HSP27 protein levels was not equivalent to that of $\mathrm{IC}_{50}$ between WiDr and HCT116. 5-FU resistance may be determined by the linkage of several molecules, which explains the lack of comprehensive results.

In addition, the protective mechanisms of HSP27 may result from its phosphorylation activity, chaperoning function or glutathione regulation (36). In our study, we did not observe the status of HSP27 phosphorylation, which may be able to clear up some of the inconsistent results described above. Further investigation regarding the relationship between HSP27 and other molecules or HSP27 phosphorylation is required to elucidate the mechanism of 5-FU resistance in colon cancer.

In conclusion, the findings of this study showed a functional correlation between HSP27 and 5-FU resistance in vitro and suggested that HSP27 was a potentially novel determinant of 5 -FU resistance in colon cancer. Further investigation of the role of HSP27 will provide additional insights into the molecular mechanism of 5-FU resistance, as HSP27 will become one of the major targets in gene therapy for colon cancer.

\section{Acknowledgements}

We are very grateful to K. Yamamoto and Asahi Breweries, Ltd. for kindly providing the HSP27 sense vector.

\section{References}

1. Heidelberger C, Chaudhuri NK, Danneberg P, et al: Fluorinated pyrimidines, a new class of tumor-inhibitory compounds. Nature 179: 663-666, 1957.
2. Meyerhardt JA and Mayer RJ: Systemic therapy for colorectal cancer. N Engl J Med 352: 476-487, 2005.

3. Mader RM, Muller M and Steger GG: Resistance to 5-fluorouracil. Gen Pharmacol 31: 661-666, 1998.

4. Johnston PG, Drake JC, Trepel J and Allegra CJ: Immunological quantitation of thymidylate synthase using the monoclonal antibody TS 106 in 5-fluorouracil-sensitive and -resistant human cancer cell lines. Cancer Res 52: 4306-4312, 1992.

5. Aschele C, Debernardis D, Casazza S, et al: Immunohistochemical quantitation of thymidylate synthase expression in colorectal cancer metastases predicts for clinical outcome to fluorouracil-based chemotherapy. J Clin Oncol 17: 1760-1770, 1999.

6. Copur S, Aiba K, Drake JC, Allegra CJ and Chu E: Thymidylate synthase gene amplification in human colon cancer cell lines resistant to 5-fluorouracil. Biochem Pharmacol 49: 1419-1426, 1995.

7. Chu E, Lai GM, Zinn S and Allegra CJ: Resistance of a human ovarian cancer line to 5-fluorouracil associated with decreased levels of 5-fluorouracil in DNA. Mol Pharmacol 38: 410-417, 1990.

8. Zhou ZW, Wang GQ, Wan DS, et al: Relationship between dihydropyrimidine dehydrogenase (DPD) activity and toxicity of 5-FU-based adjuvant chemotherapy in colorectal cancer patients. Ai Zheng 23: 1512-1516, 2004.

9. Takechi T, Koizumi K, Tsujimoto $\mathrm{H}$ and Fukushima M: Screening of differentially expressed genes in 5-fluorouracilresistant human gastrointestinal tumor cells. Jpn J Cancer Res 92: 696-703, 2001.

10. Lindquist $S$ and Craig EA: The heat-shock proteins. Annu Rev Genet 22: 631-677, 1988

11. Schlesinger MJ: Heat shock proteins. J Biol Chem 265: 12111-12114, 1990.

12. Ovelgonne JH, Koninkx JF, Pusztai A, et al: Decreased levels of heat shock proteins in gut epithelial cells after exposure to plant lectins. Gut 46: 679-687, 2000.

13. Miyazaki T, Kato H, Faried A, et al: Predictors of response to chemo-radiotherapy and radiotherapy for esophageal squamous cell carcinoma. Anticancer Res 25: 2749-2755, 2005.

14. Ciocca DR and Calderwood SK: Heat shock proteins in cancer: diagnostic, prognostic, predictive, and treatment implications. Cell Stress Chaperones 10: 86-103, 2005.

15. Ciocca DR, Fuqua SA, Lock-Lim S, Toft DO, Welch WJ and Mcguire WL: Response of human breast cancer cells to heat shock and chemotherapeutic drugs. Cancer Res 52: 3648-3654, 1992.

16. Garrido C, Ottavi P, Fromentin A, et al: HSP27 as a mediator of confluence-dependent resistance to cell death induced by anticancer drugs. Cancer Res 57: 2661-2667, 1997.

17. Garrido C, Bruey JM, Fromentin A, Hammann A, Arrigo AP, Solary E: HSP27 inhibits cytochrome c-dependent activation of procaspase-9. FASEB J 13: 2061-2070, 1999.

18. Garrido C, Mehlen P, Fromentin A, et al: Inconstant association between 27-kDa heat-shock protein (Hsp27) content and doxorubicin resistance in human colon cancer cells. Eur J Biochem 237: 653-659, 1996

19. Vargas-Roig LM, Gago FE, Tello O, Aznar JC and Ciocca DR: Heat shock protein expression and drug resistance in breast cancer patients treated with induction chemotherapy. Int J Cancer 79: 468-475, 1998.

20. Suganuma K, Kubota T, Saikawa Y, et al: Possible chemoresistance-related genes for gastric cancer detected by cDNA microarray. Cancer Sci 94: 355-359, 2003.

21. Yamauchi $\mathrm{T}$, Watanabe $\mathrm{M}$, Hasegawa $\mathrm{H}$, et al: The potential for a selective cyclooxygenase-2 inhibitor in the prevention of liver metastasis in human colorectal cancer. Anticancer Res 23: 245-249, 2003.

22. Yamamoto K, Okamoto A, Isonishi S, Ochiai K and Ohtake Y: Heat shock protein 27 was up-regulated in cisplatin resistant human ovarian tumor cell line and associated with the cisplatin resistance. Cancer Lett 168: 173-181, 2001.

23. Hwang PM, Bunz F, Yu J, et al: Ferredoxin reductase affects p53-dependent, 5-fluorouracil-induced apoptosis in colorectal cancer cells. Nat Med 7: 1111-1117, 2001.

24. Rigas A, Dervenis C, Giannakou N, Kozoni V, Shiff SJ and Rigas B: Selective induction of colon cancer cell apoptosis by 5-fluorouracil in humans. Cancer Invest 20: 657-665, 2002.

25. Voboril R, Hochwald SN, Li J, et al: Inhibition of NF-kappa B augments sensitivity to 5-fluorouracil/folinic acid in colon cancer. J Surg Res 120: 178-188, 2004. 
26. Korsmeyer SJ: Bcl-2 initiates a new category of oncogenes: regulators of cell death. Blood 80: 879-886, 1992.

27. Miyashita T and Reed JC: Bcl-2 oncoprotein blocks chemotherapy-induced apoptosis in a human leukemia cell line. Blood 81: 151-157, 1993.

28. Violette S, Poulain L, Dussaulx E, et al: Resistance of colon cancer cells to long-term 5-fluorouracil exposure is correlated to the relative level of $\mathrm{Bcl}-2$ and $\mathrm{Bcl}-\mathrm{X}(\mathrm{L})$ in addition to $\mathrm{Bax}$ and p53 status. Int J Cancer 98: 498-504, 2002.

29. Sun Y, Tang XM, Half E, Kuo MT and Sinicrope FA: Cyclooxygenase-2 overexpression reduces apoptotic susceptibility by inhibiting the cytochrome c-dependent apoptotic pathway in human colon cancer cells. Cancer Res 62: 6323-6328, 2002.

30. Kluck RM, Bossy-Wetzel E, Green DR and Newmeyer DD: The release of cytochrome $\mathrm{c}$ from mitochondria: a primary site for $\mathrm{Bcl}-2$ regulation of apoptosis. Science 275: 1132-1136, 1997.

31. Li P, Nijhawan D, Budihardjo I, et al: Cytochrome c and dATPdependent formation of Apaf-1/caspase- 9 complex initiates an apoptotic protease cascade. Cell 91: 479-489, 1997.
32. Green DR and Reed JC: Mitochondria and apoptosis. Science 281: 1309-1312, 1998.

33. Martinou JC, Desagher S and Antonsson B: Cytochrome c release from mitochondria: all or nothing. Nat Cell Biol 2: E41-E43, 2000.

34. Konishi T, Sasaki S, Watanabe T, Kitayama J and Nagawa H Overexpression of hRFI inhibits 5-fluorouracil-induced apoptosis in colorectal cancer cells via activation of NF-kappaB and upregulation of BCL-2 and BCL-XL. Oncogene: 2006.

35. Concannon CG, Orrenius S and Samali A: Hsp27 inhibits cytochrome c-mediated caspase activation by sequestering both procaspase-3 and cytochrome c. Gene Expr 9: 195-201, 2001.

36. Filomeni G, Aquilano K, Rotilio G and Ciriolo MR: Antiapoptotic response to induced GSH depletion: involvement of heat shock proteins and NF-kappaB activation. Antioxid Redox Signal 7: 446-455, 2005. 